\title{
Sostenibilidad - Recursos Naturales y su relación con la Sociedad Posmoderna en Guanacaste, Costa Rica
}

\author{
Ricardo A. Morataya Montenegro ${ }^{1}$, Juan Carlos Villegas Arguedas ${ }^{2}$ \& Steven Oreamuno Herra ${ }^{3}$ \\ 1. Universidad Nacional (UNA), Sede Regional Chorotega - ecomosa@gmail.com \\ 2. Ministerio de Ambiente y Energía (MINAE), Regional Golfito - Zona Sur - jcvilleg@gmail.com \\ 3. Universidad Nacional (UNA), Campus Omar Dengo - Decano Escuela de Topografía - \\ steven.oreamuno.herra@una.cr
}

Recibido: 04 junio 2013

Aceptado: 12 julio 2013

\section{RESUMEN}

El presente artículo hace una discusión crítica entre la sociedad postmoderna guanacasteca y su vinculación con los recursos con que cuenta. Esta relación se enfoca en tres sentidos: a) flora y fauna, b) recurso hídrico y c) urbanismo. Esto debido a que los recursos naturales de la región guanacasteca entran a jugar un papel importante para el desarrollo de la sociedad que se ha establecido desde mediados del siglo XX, dándose algunas dinámicas político-agrarias, y la consecuente destrucción "invisible" de los recursos naturales, siendo el indicador detonante de esa política la escases de agua en la región. Elartículo describe a los recursos de flora y fauna y esa relación con la conservación y turismo, la cual busca hoy en día un equilibro sustentado el fortalecimiento del turismo rural, pero el crecimiento urbano y rural en cuanto a la interacción con el medio que le rodea aumentan la demanda por el recurso agua de forma integral (producción de bienes y consumo en el hogar).

Palabras clave: sostenibilidad, recursos naturales, flora, fauna, recursos hídricos, agua, urbanismo, sociedad postmoderna.

\footnotetext{
ABSTRACT

This article makes a critical discussion between Guanacaste postmodern society and its relationship to the resources available. This relationship is focused in three ways: a) flora and fauna, b) water resources c) planning. This is because the natural resources of the Guanacaste region begin to play an important role for development of society that has been established since the mid twentieth century, giving some agrarian political dynamics, and the consequent destruction "invisible" resource natural, being the trigger for the policy indicator water scarcity in the region. The article describes the wildlife resources and the relationship with the conservation and tourism, which seeks a balance today, supported the strengthening of rural tourism, but the urban and rural growth in terms
}

of interaction with the environment that surrounding increase demand for water resources in an integrated manner (production of goods and household consumption).

Keywords: sustainability, natural resources, flora, fauna, water resources, water, urban, postmodern society.

\section{Introducción}

Guanacaste ha sufrido una transformación desde los años 40 del siglo pasado en promedio, ya que en esos años, esta tierra no muy poblada y llena de mucha vegetación exuberante, ríos abundantes, y pobladores nativos, tal como lo son los descendientes de la cultura chorotega, y que habitan principalmente Nicoya, Santa Cruz y otras bajuras. Sin embargo, en esa época se da una migración desde zonas como San Ramón, Palmares, Alajuela principalmente, a poblar zonas con potencial productivo, a donde algunas de ellas son Carmona, Tilarán, Hojancha principalmente, y a quienes se les denomina "cartagos" por no tener el estereotipo guanacasteco, sino que todo lo contrario (tez blanca, ojos claros en su mayoría, y con otras costumbres al estilo de la meseta central).

Morataya (2011), manifiesta que la dinámica ambiental de la Península de Nicoya, enfocada principalmente en la presencia o ausencia de cobertura boscosa (indicador ecológico), se puede denotar históricamente que es uno de los factores más importantes que han afectado o beneficiado la cobertura forestal de esta zona, siendo una de ellas las políticas económicas del 
Estado, a donde de una manera indirecta, se promovió la deforestación, argumentando el Estado que los agricultores debían darle valor a sus parcelas de trabajo convirtiéndolas en pastizales para ganado, de lo contrario no eran sujetos de créditos e incentivos (años 40 del Siglo $\mathrm{XX}$ en promedio) para favorecer así un mercado internacional que demandaba carne de res en esos años. Posteriormente se cae el mercado de la carne en Centro América, debido a que gigantes del cono sur de América (Argentina y Brasil principalmente) empezaron a producir más a menor precio. Esto último desfavoreció a muchos ganaderos, quienes abandonaron o vendieron sus tierras y se regresaron a sus lugares de origen, lo cual afectó económicamente a dichas familias, pero a la vez favoreció que las tierras deforestadas para ganado se recuperaran en un proceso de sucesión vegetal hasta formar de nuevo bosques, los cuales ya no iban a ser primarios sino bosques secundarios.

Hoy en día, los bosques de la Península de Nicoya, en su mayoría son bosques secundarios, los cuales emergieron después que se dio la coyuntura de la caída de los precios de la carne y abandono de tierras, dándose ese proceso de recuperación de bosques, lo cual a la postre favoreció el surgimiento de otro motor económico para Guanacaste, el cual es el turismo.

La interrogante del problema que se plantea para elaborar el presente artículo, está referida en saber ¿cuál es la dinámica actual de Guanacaste en su desarrollo integral con respecto a los recursos naturales de Flora y Fauna, acceso al agua y desarrollo urbano? El objetivo del ensayo es describir la dinámica entre recursos naturales y la sociedad guanacasteca, de tal manera que se analiza el papel de la flora y fauna como un plus base para la conservación y turismo, el comportamiento de los recursos hídricos y la vinculación de la sociedad y el ambiente, enmarcándose todo en el concepto de desarrollo sostenible.
Así también, es importante entender lo que es sostenibilidad, cuyo punto de partida fue en 1987 con el informe Brundtland, el cual entre otras cosas, indica que "Desarrollo Sostenible", se refiere a "Satisfacer las necesidades de las generaciones presentes sin comprometer las posibilidades de las del futuro para atender sus propias necesidades". A donde indican también que el desarrollo sostenible se refiere a la totalidad de las actividades humanas, y por otro lado, los retos de la sostenibilidad, son diferentes para cada tipo de sector económico, tal como la Agricultura, Industria y Servicios principalmente. El concepto de sostenibilidad se refiere más a la dinámica y disponibilidad del recurso en cuestión, o sea, que se refiere a cuan perdurable es el recurso. La Comisión Mundial de Ambiente y Desarrollo plantea que el desarrollo sostenible "es aquel desarrollo que satisface las necesidades del presente sin comprometer la habilidad de las futuras generaciones para satisfacer sus propias necesidades". La pregunta obvia que se genera es si esa visión futurista de las necesidades futuras se convierte en una posición egoísta, ya que las actuales también tienen necesidades.

Por lo tanto, el documento aborda esas dinámicas, donde se han favorecido la conservación y por otro lado, se ha incentivado el turismo en función de esa conservación, pero lo que también surge es que todo ese movimiento social provoca sinergias, las cuales empiezan a enraizar en un crecimiento urbano y comercial de las zonas costeras primeramente, y luego el crecimiento normal de la población, pero que ya en su conjunto ofrece una demanda de servicios y recursos en constante aumento, siendo el más vulnerable la "disponibilidad de agua" para sustentar la misma. Tal es el caso actual de los crecimientos urbanos en zonas como Liberia, Santa Cruz y Nicoya entre otras ciudades principalmente, lo cual aumenta la demanda de recursos, principalmente agua. 
Sin embargo, tal como lo indica Buska (2006), el pueblo guanacasteco ha engrandecido a la nación costarricense, no solo en territorio, sino en valores, cultura e intelectualidad. Desde albores de la historia del "Partido de Nicoya" como parte del territorio de Costa Rica. Esta sociedad ha demostrado lealtad y patriotismo, pujando fuertemente por el desarrollo de su provincia y la reivindicación de sus derechos por ser una provincia que ha contribuido con la identidad nacional, a pesar que en muchas ocasiones el Estado no ha cumplido con su deber social de procurar un desarrollo sostenible para con estos compatriotas.

En adelante, se desarrollan tres frentes de discusión, para así relacionar la sociedad posmoderna actual y el papel que juegan para con los recursos naturales (flora y fauna), recursos hídricos y la interacción urbanismo y ambiente. Todo analizado desde la perspectiva de la sostenibilidad.

\section{Análisis coyuntural}

Flora y fauna: conservación para el desarrollo

Esta región pacífico-norte ha pasado por distintas etapas en la conservación de sus recursos naturales, desde casi desaparecer su cobertura forestal por la apertura de claros para el pastoreo de los hatos ganaderos (Gómez 1989, Stoner y Timm 2004), hasta convertirse en la actualidad en una de las provincias con mayor recuperación y restauración de bosques, los cuales se encuentran en franca recuperación desde hace unos 30 años, visibles en distintos estudios geoespaciales sobre el tema (Calvo et al. 1999, Calvo y Ortiz 2012), bosques que con el pasar del tiempo ganan diversidad faunística y arbórea (Elizondo y Jiménez 1988, Leiva et al. 2009).

La recuperación de estos ecosistemas ha traído consigo también la recuperación de la fauna, la cual había tenido como único refugio las Áreas Silvestres Protegidas que el Estado ha promovido y establecido en la provincia, previo a la actual conformación de los nuevos corredores biológicos. Gracias a esta ampliación de los bosques, Guanacaste hoy día cuenta con registros de al menos 78 especies entre anfibios y reptiles (Laurencio 2009), alrededor de 155 especies de mamíferos (Rodríguez y Chinchilla 1996), de los cuales son propios del bosque seco 114 (Stoner y Timm 2004), unas 300 especies de aves (Jansen 1991) entre muchas otras bellezas naturales.

Son varios los esfuerzos que se han desarrollado en materia de recuperación de bosques en la región de Guanacaste, esfuerzos que no solo han aumentado la cantidad de ecosistemas naturales, sino que han beneficiado a la fauna local, Mello et. al. (2010) reportaron como a partir de su programa de recuperación de los bosques en las partes medias y altas del bosque nuboso en Monteverde, lograron después de ocho años avistar monos congo (Allouatta palliata) en sus bosques recuperados; otras iniciativas se dieron en el mismo periodo en las partes bajas, especialmente recuperando zonas de protección de ríos y quebradas (Gómez 1989).

Las riquezas naturales guanacastecas no se quedan solo en el bosque seco, hacia la costa y en la plataforma continental marina se encuentran ecosistemas muy importantes y biodiversos. Zamora y Cortés (2009) han recopilado mucha información de las áreas de manglar del pacífico norte, las cuales conservan poblaciones de especies que en el pasado fueron diezmadas drásticamente por el consumo local como las pianguas y las chuchecas (Anadara tuberculosa, Anadara multicostata, Crassostrea corteziensis y Grandiarca grandis) además de gasterópodos como Littorina zebra y Cerithidea valida, y otros grupos animales como los crustáceos de los géneros Uca y Penaeus.

Otros datos interesantes son los estudios sobre los ratones del manglar Oryzomys palustris, roedor que se captura con mayor frecuencia, mientras que Sigmodon hispidus también se distribuye en estos manglares pero en menor 
cantidad (Zamora y Cortés 2009). Estos mismos autores reportan avifauna importante como Phalacrocorax olivaceus (pato aguja), Fregata magnificens (zopilotillo de mar), Buteogallus anthracinus (gavilán cangrejero), Buteo magnirostris (gavilán chapulinero) y Pandion hallaetus (tijerillo), y los mamíferos: Vampirum spectrum (murciélago gigante) y Didelphis marsupiales (zorro pelón o zariguella) así como monos y lagartos (Caiman crocodrilus fuscus).

Hoy día la actividad socio productiva más importante de la provincia guanacasteca es el turismo (Morales 2009) y no cabe duda que la conservación de estos recursos naturales juega un papel fundamental para esta actividad y su sostenibilidad financiera, lo que viene a ser un buen ejemplo de desarrollo sostenible donde tanto el recurso natural se conserva y la economía familiar se beneficia con nuevos y mejores empleos.

Hay muchos ejemplos que testifican esta buena relación turismo-conservación, de los cuales es posible enumerar algunos datos interesantes sobre el beneficio de esta simbiosis para el desarrollo sostenible de esta región, Almeyda et al. (2010) enumeran una serie de indicadores sobre mejora en la calidad ambiental y social en la zona del desarrollo turístico "Punta Islita" y la cadena de valores que se generaron a raíz del mismo. Este es un proyecto eco turístico que se desarrolla en la Península de Nicoya en antiguas tierras que fueron deforestadas para ganadería hacia la década de 1940.

Datos sobre la cobertura forestal en las cercanías del proyecto indican que aumentó de manera importante y sostenida desde su establecimiento, a su vez se cuantificó la inversión considerable que los turistas están dispuestos a realizar por permanecer en el sitio; otras percepciones entre las comunidades y empleados de "Punta Islita" indican que la cacería y los delitos ambientales han disminuido drásticamente gracias al turismo en la región, además del aumento del empleo y la educación de las personas que trabajan para el proyecto y las cercanías (Almeyda et al 2010).

No se puede olvidar que el Estado también ha hecho esfuerzos para coadyuvar en la conservación de importantes ecosistemas guanacastecos, administrados y resguardados como Patrimonio Natural del Estado en las Áreas de Conservación Guanacaste y Tempisque, de las cuales los costarricenses y en especial los guanacastecos pueden vanagloriarse de contar con el Parque Nacional Santa Rosa, el PN. Guanacaste, el PN. Marino Las Baulas, PN. Rincón de la Vieja, PN. Barra Honda, PN. Palo Verde, el Refugio de Vida Silvestre Bahía Junquillal, el RVS. Ostional y la Reserva Natural Absoluta Cabo Blanco.

\section{Recursos hídricos: retos y desafíos}

Antes de discutir los retos y desafíos que enfrentará Guanacaste en este 2013 y en adelante, es importante tener una panorámica general en Costa Rica, donde hoy en día, muchas no cuentan con dicho acceso, lo cual representa, según el Programa Estado de la Nación (2010) un 10,5\%, es decir; que de cada 10 costarricenses 1 no está recibiendo agua potable, lo que indica que en realidad podría ser una población promedio de 451500 habitantes en todo el país sin acceso al agua potable, considerando que según el último informe de población la misma llegó a 4.3 millones de habitantes (Instituto Nacional de Estadística y Censo -INEC-, 2011). Por otro lado, la proporción de habitantes sin el servicio de agua potable mencionados anteriormente, suelen tener otras alternativas, tales como: una conexión directa desde algún naciente aguas arriba, pozos artesanales, o directamente desde un río cercano, la cual, en mucho de los casos debe ser acarreada por sistemas manuales que requieren de la fuerza humana, tales como baldes plásticos, y otros afines. Por otro lado, según el mismo informe del Programa Estado de la Nación (2010), indica que del $100 \%$ de 
Asociaciones Administradoras de Acueductos Rurales (ASADA) que tiene todo el país de Costa Rica (un total 1067 Asadas), se determinó que 465 Asadas $(43,6 \%)$ no suministran agua potable. Todo lo anterior puede dar a confusiones, ya que se indica que un $10,5 \%$ de la población total de Costa Rica no tiene acceso al agua, y del total de Asadas, un poco menos de la mitad distribuyen agua a las comunidades rurales pero en condición no potabilizada. Esto puede limitar a los pobladores rurales de tener agua de calidad y cantidad, y que en sí, puede repercutir directamente en la calidad de vida, principalmente el rubro de salud, que a la postre, podría desencadenar un "efecto dominó" sobre otras actividades, que de una u otra manera podrían favorecer un desarrollo integral de las comunidades. En síntesis, una comunidad enferma o limitada, no podrá, en teoría, propiciarse un desarrollo integral.

Guanacaste actualmente tiene varios retos y desafío a afrontar, los cuales de no resolverse, atentan contra la sostenibilidad de dicho recurso, el cual es de vital importancia para el desarrollo de la vida en su contexto general. Sin embargo, antes de adentrarse en los desafíos, es importante denotar las cuatro áreas críticas que fueron detectadas por Kuzdas (2012):

1. Existe una falta de coordinación e intercambio de información entre comunidades sobre las extracciones de agua en los acuíferos principales de Guanacaste. Esto genera una incertidumbre en cuanto a la contabilidad de la cantidad de agua que se usa y la que se dispone, y los Balances Hídricos no están a la orden de los administradores del servicio de agua, tales como las Asociaciones Administradoras de Acueductos Rurales (ASADA) principalmente y el mismo Instituto Costarricense de Acueductos y Alcantarillados (AyA), que en éste último caso no se tiene la confianza de que se basen en dicha contabilidad para asignar servicios de agua, lo cual ha causado colapsos en Guanacaste (asignación de servicios en zonas costeras sin prever el abastecimiento). Todo lo contrario sucede con las ADASA's, las cuales al no tener a la mano un informe científico sobre lo que cuentan (balance hídrico actualizado permanentemente), entonces no tienen un argumento legal para no dar más asignaciones más allá del potencial de agua actual, lo cual es una realidad para el Acueducto de Nosara. En esta comunidad, las ASADAS han tenido que dar agua sin tenerla a hoteles y similares, ya que la ley los obliga a prestar el servicio.

2. Hay poca integración y comunicación entre comunidades que habitan las partes altas, medias y bajas de las cuencas en Guanacaste, y esta falta de coordinación hace que las comunidades se vean afectadas entre sí. Uno de los casos entre otros, son las ciudades de Liberia, Santa Cruz y Nicoya. En Liberia se sabe que de acuerdo a datos del AyA, que el $60 \%$ a $70 \%$ del agua que usan los Liberianos procede del río Liberia, el cual en su parte media atraviesa la misma ciudad, pero en su salida y trayecto antes de llegar al río Tempisque, el río se convierte en una cloaca, la cual parece transparente, pero lleva basura y vertidos que la misma ciudad de Liberia vierte en el mismo. Por otro lado, en la ciudad de Santa Cruz sucede lo mismo con el río En Medio, y también para Nicoya sucede con dos ríos que atraviesan esta ciudad, y que los tres forman al final el río Grande o Morote. Estos ríos son el río Perico y Cinpanci, los cuales atraviesan Nicoya Centro, y al final también forman el río Morote. Pero antes, al final de la ciudad de Nicoya están las lagunas de oxidación, las cuales son muy mal manejadas, y el exceso putrefacto que se genera es depositado por gravedad al río Grande o Morote. Lo duro y real, es ver en el Pueblo de Mansión (unos 10 km río debajo de Nicoya), a niños y niñas jugando en dicho río y disfrutando de sus aguas (contaminadas).

3. Se presenta una total ausencia de liderazgo que coordine actividades para la educación 
del uso del agua. Así también indica que hay una ausencia de un norte común en los esfuerzos de manejo. Esto último se evidencia en las reuniones que tratan sobre el recurso hídrico, a donde el AyA nunca o casi nunca, es un protagonista. Esto ha sido la realidad en Guanacaste.

4. Se evidencia que hay reglas inadecuadas de regulación y monitoreo del uso del agua, y esto es especialmente después de que el agua ya ha sido utilizada.

Es importante considerar con seriedad la reincorporación de este preciado líquido a la naturaleza, ya que también es un factor de desarrollo integral, debido que su manejo residual adecuado puede en sí desentonar el tan ansiado desarrollo social y económico que esta región requiere. Por lo tanto, si no existe un sistema claro que permita a los usuarios del agua potable devolver a la naturaleza la misma debidamente tratada (con capacidad de sustentar la vida nuevamente), esta se convertirá en un obstáculo grande que se debe enfrentar con prontitud, ya que actualmente hay muchos pueblos que aún hoy en día, tiran las aguas jabonosas al caño de la calle.

Con base a lo expuesto anteriormente, Guanacaste tiene varios desafíos actuales, siendo:

1. Problemas de abastecimiento de agua hoy en día.

2. Gobierno con mucha burocracia en temas de agua, siendo desgastante y asfixiante.

3. Cambio climático con evidencias reales de su presencia hoy en día.

4. Falta una sistematización e integración de información para las cuencas de la región, de tal manera que se pueda dar un enfoque ecosistémico en la dinámica de sus componentes.

5. Debe haber un solo líder en agua. Ya que actualmente hay muchas instituciones que se encargan del recurso hídrico (AyA, MINAET, SENARA, Ministerio de Salud, etc.).
6. Continuo crecimiento urbano.

7. Incendios forestales y quemas agrícolas no controladas.

8. Crisis económica actual del país y sus repercusiones en la dinámica comercial de Guanacaste, principalmente el turismo y los recursos naturales.

9. El mayor desafío, es generar una cultura de un buen manejo y uso eficiente del agua por parte de la ciudadanía usuaria del mismo, ya sean hogares, pequeñas empresas, agro industrias, etc.

Urbanismo: lo que ayer era sostenible hoy no lo es, la población crece.

Décadas antes, por decir de dos a tres, lugares rurales de Guanacaste no sufrían de problemas de aguas negras, sino que todo se solucionaba con el hoyo negro o excusado, y también los desechos líquidos se volvían a infiltrar. Posiblemente ese escenario anterior hacía sostenible el proceso, en virtud que la velocidad de descomposición y de infiltración era tal, que al llegar a reintegrarse el agua con los cursos normales, ésta ya llegaba de nuevo limpia. Sin embargo, al superarse la velocidad de desechos al ambiente con respecto a su asimilación se provoca lo que hoy vemos como contaminación visible. En adelante se analiza ese cambio a través del tiempo.

Todos los que viven en Guanacaste son privilegiados por tener la fortuna de vivir en tierras con tanta belleza natural, con sus áreas protegidas, cantidad de ríos y sin olvidar sus maravillosas playas, sin embargo, durante los últimos años se ha venido dando un acelerado crecimiento urbano sin ninguna planificación territorial y mucho más en las zonas costeras de esa provincia.

Básicamente los desarrollos urbanísticos como los son los condominios horizontales o urbanizaciones en donde los propietarios construyen sus casas individualmente y los condominios verticales, edificios de varios pisos con campos de golf, piscinas, zonas verdes 
entre otros, son los factores que desde el punto de vista económico y comercial puede resultar positivos pero desde el punto de vista ambiental tienen muchos efectos que deben ser considerados de forma integral y previstos de manera apropiada para evitar efectos negativos en el ambiente.

Con respecto en el tema social, el cambio en la dinámica cultural y sus patrones, así como la presión por los servicios, tanto en cantidad como en calidad y su consecuente efecto en los precios, son solo algunos de los aspectos que deben ser tomados en cuenta como parte del análisis integral de este tipo de desarrollo, cosa que no se da responsablemente.

En todo proyecto urbanístico de cualquier índole hay requisitos que deben cumplirse como lo es: la disposición de agua potable, no solo para la construcción, sino también para la operación de las residencias. Si el proyecto se encuentra en una zona donde existen árboles, o zonas boscosas, se necesita el permiso de tala de árboles ante el MINAE fuera los permisos normales del Colegio Federado de Ingenieros y de Arquitectos, así como el de la Municipalidad, Ministerio de Salud, Instituto Nacional de Vivienda y Urbanismo (INVU), Acueductos y Alcantarillados (AyA), ICE o las entidades encargadas de proveedor la energía eléctrica y el agua potable. Además, uno de los trámites más importantes es el de la Viabilidad Ambiental ante la Secretaría Técnica Ambiental (SETENA) y que no se visualiza en su dimensión real, ya que se interpreta por la gran mayoría de usuarios e incluso funcionarios públicos como un requisito más de la lista de trámites que debe cumplir un proyecto de desarrollo urbanístico. No obstante, la Viabilidad Ambiental, obtenida mediante la Evaluación de

Impacto Ambiental (EIA) es una cosa completamente diferente y de gran trascendencia, ya que cuando se otorga el permiso de Viabilidad Ambiental de un proyecto sea cual sea, esta entidad SETENA está firmando que el mismo se podrá ejecutar porque se ha analizado y concluido que no generará impactos significativos al ambiente, no solo durante su construcción, sino también durante su operación.

Según lo ha interpretado la Sala Constitucional, la Viabilidad Ambiental de un proyecto quiere decir que se está afirmando, tácitamente, que el proyecto puede ser realizado en el marco de que se garantiza el cumplimiento de lo establecido en el Artículo 50 de la Constitución Política de Costa Rica que dice textualmente "El Estado procurará el mayor bienestar a todos los habitantes del país, organizando y estimulando la producción y el más adecuado reparto de la riqueza. Toda persona tiene derecho a un ambiente sano y ecológicamente equilibrado. Por ello, está legitimada para denunciar los actos que infrinjan ese derecho y para reclamar la reparación del daño causado. El Estado garantizará, defenderá y preservará ese derecho. La ley determinará las responsabilidades y las sanciones correspondientes. (Así reformado por el artículo $1^{\circ}$ de la ley No.7412 de 3 de junio de 1994)"

Por ende, se puede decir que un proyecto urbanístico genera un impacto ambiental pequeño y que puede ser controlable, pero si ya no es uno, sino dos, tres o muchos proyectos en una misma zona como esta sucediendo en Guanacaste, se producen daños al ambiente difícil de controlar y como ejemplos se puede citar el uso irracional del agua subterránea para mantener esas grandes extensiones de terrenos destinados a campos de golf, jardines o piscinas de los condominios, urbanizaciones o grandes hoteles principalmente en la zona costera, produciendo una sobreexplotación de los mantos acuíferos impactando en el ambiente, así como en los servicios de energía, recursos minerales, drenaje de aguas pluviales, vertidos de aguas residuales, manejo de residuos sólidos ordinarios y especiales, recursos forestales, incidencias sociales, servicios de salud, recursos culturales, recursos biológicos terrestres y 
marinos, condiciones de vulnerabilidad a las amenazas naturales y recursos paisajísticos, entre otros.

\section{Conclusiones y recomendaciones}

Se puede afirmar que en la provincia de Guanacaste han sido grandes y muchos los esfuerzos por desarrollar sosteniblemente la zona, son muchos los buenos ejemplos de actividades socioproductivas que contemplan la responsabilidad social y ambiental en su quehacer, y se ha demostrado con el aumento de la cobertura forestal y la recuperación de la fauna que son muchas las buenas intenciones. Pero también sigue siendo evidente que hay otras cosas que empañan estos buenos esfuerzos; año a año se continúa quemando el bosque seco tropical por personas inescrupulosas, en otras ocasiones por cazadores, los cuales continúan haciendo daño a la fauna local.

Lamentable y antagónicamente con los esfuerzos de conservación y desarrollo sostenible, en la región se continúan desarrollando actividades ilícitas y no compatibles con la armonía natural; de las 114 especies de mamíferos registradas en el bosque seco tropical, Stoner y Timm (2004) mencionan que es posible que en la actualidad solo se conserven 110 debido a la desaparición por cacería o por estrés ecológico del pasado por los agresivos procesos de deforestación, los mismos autores mencionan que después de 1880 la mayoría de pieles de venado cola blanca que se exportaron a Europa fueron del bosque seco del pacífico norte, problema que continua en las áreas protegidas en el Área de Conservación Guanacaste y el Área de

Conservación Tempisque. No obstante el bosque ha regresado a muchas zonas de la provincia, aún en esta región éste es considerado en peligro o bajo riesgo de desaparecer en el futuro (Bonilla y Rosero 2004), dependiendo del rumbo que el crecimiento de los pueblos o eventuales urbanizaciones costeras desarrollen, lo cual es una alerta temprana para no abandonar las buenas acciones sostenibles que ya rinden sus frutos a esta patriótica provincia guanacasteca.

En la temática de agua, Guanacaste aparte de ser una zona naturalmente con una estación seca marcada, evidencia un desbalance en la disponibilidad de agua, tanto para consumo como para uso agroindustrial y empresarial (pequeña, mediana y gran empresa). Este desbalance en su disponibilidad se ve con claridad durante este verano recién pasado, a donde muchos ríos que anteriormente no se había secado su caudal ecológico, en esta ocasión si sucedió.

Es evidente, con los estudios mostrados y con la experiencia presencial en Guancaste, de que se carece de un norte común en cuanto a lo que es el uso y manejo del agua, ya que existe una desconcentración de acciones, a donde varias instancias gubernamentales se les asigna responsabilidad. Esto demuestra una extrema debilidad organizacional para afrontar los nuevos retos que se avecinan con posibles altibajos del recurso hídrico.

Es imperante que Guanacaste se inicie a adaptar a condiciones extremas para sobrevivir (resiliencia), de tal manera que exista una adaptación natural a las nuevas condiciones que se derivan del cambio climático.

En la zona de Guanacaste el desarrollo de proyectos urbanísticos ha traído beneficios como más divisas y empleos a sus habitantes, pero también ha llevado bastantes daños ambientales, en donde se debe de tener cuidado para que esos daños no sigan avanzando y se debe mencionar, que se han hecho grandes esfuerzos para atender esta situación (incorporación de la variable ambiental en los planes reguladores, mejora de los instrumentos como el D-1, etc.), no obstante, hacen falta soluciones más integrales y duraderas.

Esas soluciones deben de ser implementadas a muy corto plazo, ya que la velocidad y aceleración del crecimiento urbano con poca planificación o con una planificación 
irresponsablemente avanza cada día más y nos quedamos con menos recursos naturales en esa zona, por lo que es urgente que esta problemática sea atendida de una forma integral por parte del gobiernos y en particular por el Ministerio del Ambiente y Energía, órgano rector en materia de medio ambiente, gestión ambiental, manejo de recursos naturales de tipo forestal, energía, minero y de recursos hídricos; y también de ordenamiento ambiental territorial, claro está! con la colaboración de todos los individuos que habitan en la provincia de Guanacaste y el país en general.

\section{Referencias}

ALMEYDA, A.; BROADBENT, E.; WYMAN, M. \& DURHAM, W. (2010). Ecotourism impacts in the Nicoya Peninsula, Costa Rica. Int. J. Tourism Res. 12, 803-819.

BONILLA, R. Y ROSERO-BIXBY, L. (2004). Presión demográfica sobre los bosques y áreas protegidas, Costa Rica 2000. En Rosero Bixby, L. (Editor). Costa Rica a la luz del Censo del 2000. San José, Costa Rica: Centro Centroamericano de Población de la Universidad de Costa Rica, Proyecto Estado de la Nación e Instituto de Estadística y Censo (Imprenta Nacional), 575-594 pp.

BUSKA, S. (2006). Guanacaste: el surgimiento de un discurso regionalista, 1900-1926. Rev. Hist. 53-54: 143-168.

CALVO, A. Y ORTIZ, E. (2012). Fragmentación de la cobertura forestal en Costa Rica durante los periodos 1997-2000 y 2000-2005. Rev. For. Mes. Kurú 9 (22): $10-21$.

CALVO, J.; WATSON, V Y BOLAÑOS, R. (1999). Estudio de coberturas forestales de Costa Rica empleando imágenes LanSat 1986/87 y 1996/97. Conferencia 96. XI Congreso de Conservación Internacional. 461466 pp. Elizondo, L. y Jiménez, Q. (1988). La sabana arbolada "El Escobio", Liberia, Guanacaste, Costa Rica. Rev. Biol. Trop. 36 (2A): 175-185.

GÓMEZ, A. M. (1989). Alternative strategies for the increases participation of women in reforestation efforts in Hojancha. Costa Rica. NWSA Journal 1 (4): 762-764.
GUZMÁN, M. (2006). El acelerado crecimiento inmobiliario en las zonas costeras de Costa Rica, el caso de Guanacaste y la necesidad de una estrategia ambiental integral para la prevención de daños al ambiente por efectos acumulativos. SETENA, Costa Rica.

INSTITUTO NACIONAL DE ESTADÍSTICA Y CENSOS -INEC. (2011). X Censo Nacional de Población y VI de Vivienda 2011: resultados generales. (Documento Electrónico).

KUZDAS, C.; WIEK, A.; WARNER, B.; VIGNOLA, R.; MORATAYA MONTENEGRO, R. 2012. Integrated and participatory analysis of regional water governance regimes: the case of Guanacaste, Costa Rica. Water Resources Management.

LAURENCIO, D. (2009). Amphibians and reptiles from Reserva Natural Absoluta Cabo Blanco, province of Puntarenas, Costa Rica. Check List 5 (3): 446-459.

LEIVA, J.; ROCHA, O.; MATA, R. Y GUTIERREZ, M. (2009). Cronología de la regeneración del bosque tropical seco en Santa Rosa, Guanacaste, Costa Rica. II. La vegetación en relación con el suelo. Rev. Biol. Trop. 57 (3): 817-836.

MELLO, A.; TOWNSEND, P. \& FILARDO, K. (2010). Reforestation and restoration at the cloud forest school in Monteverde, Costa Rica: Learning by doing. Rev. Ecol. Rest. 28 (2):148-150.

MORALES, L.C. (2009). Santa Cruz de Guanacaste: Cultura local, turismo y globalización. Rev. Cienc. Social. 123-124 (I- II): 35-48.

MORATAYA MONTENEGRO, R. 2011. Dinámica ambiental actual de la Península de Nicoya. (RE) Lecturas de Guanacaste: 1821 - 2010. Universidad de Costa Rica (UCR).

PROGRAMA ESTADO DE LA NACIÓN, (2010). Estado de la Nación 2010: Sinopsis. (Documento Electrónico).

RAMÍREZ, A. (2006). Conflictos socio ambientales y recursos hídricos en Guanacaste; una descripción desde el cambio en el estilo de desarrollo (1997-2006). Anuario de Estudios Centroamericanos, Universidad de Costa Rica

RODRÍGUEZ, J. Y CHINCHILLA, F. (1996). Lista de mamíferos de Costa Rica. Rev. Biol. Trop., 44(2): 877-890. 
SENARA (2003). Diagnóstico de recursos hídricos subterráneos de la zona Guanacaste-sector norte. San José: Servicio Nacional de Aguas Subterráneas, Riego y Avenamiento, 2003a; SENARA. Diagnóstico de recursos hídricos subterráneos de la zona Guanacastesector sur. San José: Servicio Nacional de Aguas Subterráneas, Riego y Avenamiento.

STONER, K. E. \& TIMM, R. M. (2004). Tropical dry-forest mammals of Palo Verde: Ecology and conservation in a changing landscape. Pp. 48-66, in Biodiversity conservation in Costa Rica: Learning the lessons in a seasonal dry forest (G. W. Frankie, A. Mata, and S. B. Vinson, eds.). University of California Press, Berkeley, 341 pp.

ZAMORA, P. Y CORTÉS, J. (2009). Los manglares de Costa Rica: el Pacífico norte. Rev. Biol. Trop. 57 (3): 473-488. 